\title{
Compressed sensing of approximately sparse signals
}

\author{
Mihailo Stojnic \\ School of Industrial Engineering \\ Purdue University \\ West Lafayette IN 47907, USA \\ Email: mstojnic@purdue.edu
}

\author{
Weiyu Xu \\ Department of Electrical Engineering \\ California Institute of Technology \\ Pasadena CA 91125, USA \\ Email: weiyu@systems.caltech.edu
}

\author{
Babak Hassibi \\ Department of Electrical Engineering \\ California Institute of Technology \\ Pasadena CA 91125, USA \\ Email: hassibi@systems.caltech.edu
}

\begin{abstract}
It is well known that compressed sensing problems reduce to solving large under-determined systems of equations. If we choose the compressed measurement matrix according to some appropriate distribution and the signal is sparse enough the $l_{1}$ optimization can exactly recover the ideally sparse signal with overwhelming probability [2], [1]. In the current paper, we will consider the case of the so-called approximately sparse signals. These signals are a generalized version of the ideally sparse signals. Letting the zero valued components of the ideally sparse signals to take the values of certain small magnitude one can construct the approximately sparse signals. Using a different but simple proof technique we show that the claims similar to those of [2] and [1] related to the proportionality of the number of large components of the signals to the number of measurements, hold for approximately sparse signals as well. Furthermore, using the same technique we compute the explicit values of what this proportionality can be if the compressed measurement matrix $A$ has a rotationally invariant distribution of the null-space. We also give the quantitative tradeoff between the signal sparsity and the recovery robustness of the $l_{1}$ minimization. As it will turn out in an asymptotic case of the number of measurements the threshold result of [1] corresponds to a special case of our result.
\end{abstract}

Index Terms: compressed sensing, $l_{1}$-optimization

\section{INTRODUCTION}

In this paper we are interested in compressed sensing problems. As is well known these problems are very easy to pose and very difficult to solve. Namely, we would like to find $\mathrm{x}$ such that

$$
A \mathbf{x}=\mathbf{y}
$$

where $A$ is an $m \times n$ measurement matrix and $\mathbf{y}$ is $m \times 1$ measurement vector. In usual compressed sensing context $\mathbf{x}$ is $n \times 1$ unknown $k$-sparse vector. This assumes that $\mathbf{x}$ has only $k$ nonzero components. In this paper we will consider a more general version of the $k$-sparse vector $\mathbf{x}$. Namely, we will assume that $k$ components of vector $\mathbf{x}$ have large magnitude and that the vector comprised of the remaining $n-k$ components has norm 1 less than $\delta$. We will refer to this type of signals as $k$-approximately sparse signals, or for brevity only approximately sparse signals. More on similar type of problems the interested reader can find in [16].

In the rest of the paper we will further assume that the number of the measurements is $m=\alpha n$ and the number of the "large" components of $\mathbf{x}$ is $k=\beta n$, where $0<\beta<1$ and $0<\alpha<1$ are constants independent of $n$. This problem setup is probably more realistic in practical applications than the standard compressed sensing of the $k$-sparse signals. In some sense it is a quite natural generalization of the standard compressed sensing of the $k$-sparse signals which arises in many practical applications (see e.g. [17], [18] and references therein).

A particular way of solving (1) which recently generated a large amount of research is called $l_{1}$-optimization [2]. It proposes solving the following problem

$$
\begin{aligned}
\min & \|\mathbf{x}\|_{1} \\
\text { subject to } & A \mathbf{x}=\mathbf{y} .
\end{aligned}
$$

Quite remarkably in [2] the authors were able to show that if the number of the measurements $m=\alpha n, \alpha$, and $n$ are given, the matrix $A$ is given and satisfies a special property called the restricted isometry property (RIP), then any unknown vector $\mathbf{x}$ with no more than $k=\beta n$ (where $\beta$ is an absolute constant which of course is a function of $\alpha$, independent of $n$, and explicitly calculated in [2]) non-zero elements can be recovered by solving (2). As expected, this assumes that $\mathbf{y}$ was in fact generated by that $\mathbf{x}$ and given to us (more on the case when the available measurements are noisy versions of $\mathbf{y}$ interested reader can find in e.g. [13], [14]).

As can be immediately seen, the previous result heavily relies on the assumption that the measurement matrix $A$ satisfies the RIP condition. What is indeed remarkable about [2] is the fact that for several specific classes of matrices the RIP holds with overwhelming probability. It happens that if the components of $A$ are i.i.d. zero mean Gaussian or Bernoulli the RIP condition holds with overwhelming probability [2], [3], [4]. However, it should be noted that the RIP is only a sufficient condition for $l_{1}$-optimization to produce a solution of (1).

Instead of characterizing the $m \times n$ matrix $A$ through the RIP condition, in [1] the authors assume that $A$ constitutes a $k$-neighborly poly-tope. It turns out (as shown in [1]) that this characterization of the matrix $A$ is in fact a necessary and sufficient condition for (2) to produce the solution of (1). Furthermore, using the results of [5], it can be shown that if the matrix $A$ has i.i.d. zero-mean Gaussian entries with overwhelming probability it also constitutes a $k$-neighborly poly-tope. Of course, the precise relation between $m$ and $k$ in order for this to happen is characterized in [1] as well. It should also be noted that for a given value $m$ i.e. for a given value 
of the constant $\alpha$, the value of the constant $\beta$ is significantly larger in [1] than in [2]. Furthermore, the values of constants $\beta$ obtained for different values of $\alpha$ in [1] approach the ones obtained by simulation as $n \longrightarrow \infty$.

However, all these results rely on the assumption that the unknown vector $\mathbf{x}$ has only $k$ non-zero components. As we have said above in this paper we will be interested in the case of $k$-approximately sparse signals. Since in this case the unknown vector $\mathbf{x}$ in general has no zeros it is relatively easy to see that its exact recovery from a reduced number of measurements is not possible. Instead, we will prove that, if the unknown $k$-approximately sparse vector is $\mathbf{x}$ and $\hat{\mathbf{x}}$ is the solution of (2) then for any given constant $0 \leq \alpha \leq 1$ there will be a constant $\beta$ such that

$$
\|\hat{\mathbf{x}}-\mathbf{x}\|_{1} \leq \frac{2(C+1) \delta}{C-1}
$$

where $C>1$ is a given constant determining how close in norm 1 the recovered vector $\hat{\mathbf{x}}$ should be to the original $k$-approximately sparse vector $\mathbf{x}$. As expected, $\beta$ will be a function of $C$ and $\alpha$. However, $\beta$ will be an absolute constant independent of $n$. A similar problem was considered with different proof technique in [16]. In this paper we will provide the explicit values of the allowable constants $\beta$.

To prove the previous statements we will make use of another characterization that guarantees $l_{1}$ optimization works for the matrix $A$. This characterization will be equivalent to the neighborly polytope characterization from [1] in the "perfectly sparse" (the unknown vector $\mathbf{x}$ has only $k$ non-zero elements) case since it also constitutes both necessary and sufficient conditions which the matrix $A$ should satisfy in order that (2) approximates the solution of (1) such that (3) holds. We will provide a simpler analysis than [1] which will show that a non-zero $\beta$ is achievable even in the case of the approximately sparse signals. Furthermore, as we will see later in the paper, in the perfectly sparse (which allows $C \longrightarrow 1$ ) and asymptotic $(\alpha \longrightarrow 1)$ case our result for allowable $\beta$ will match the result of [1].

\section{NULL-SPACE CHARACTERIZATION}

In this section we introduce a useful characterization of the matrix $A$. The characterization will establish a necessary and sufficient condition on the matrix $A$ so that solution of (2) approximates the solution of (1) such that (3) holds. (See [10], [11], [12] for variation of this result).

Theorem 1: Assume that an $m \times n$ measurement matrix $A$ is given. Further, assume that $\mathbf{y}=A \mathbf{x}$ and that $\mathbf{w}$ is an $n \times 1$ vector. Let $K$ be any subset of $\{1,2, \ldots, n\}$ such that $|K|=k$ and let $K_{i}$ denote the $i$-th element of $K$. Further, let $\bar{K}=\{1,2, \ldots, n\} \backslash K$. Then the solution $\hat{\mathbf{x}}$ produced by (2) will satisfy $\|\mathbf{x}-\hat{\mathbf{x}}\|_{1} \leq \frac{2(C+1)}{C-1}\left\|\mathbf{x}_{\bar{K}}\right\|_{1}$, with $C>1$, if and only if

$$
\left(\forall \mathbf{w} \in \mathbf{R}^{n} \mid A \mathbf{w}=0\right) \quad \text { and } \quad \forall K, \quad C \sum_{i=1}^{k}\left|\mathbf{w}_{K_{i}}\right| \leq \sum_{i=1}^{n-k}\left|\mathbf{w}_{\bar{K}_{i}}\right| .
$$

Proof: Sufficiency: Suppose the matrix $A$ has the claimed null-space property. Then the solution $\hat{\mathbf{x}}$ of (2) satisfies $\|\hat{\mathbf{x}}\|_{1} \leq$ $\|\mathbf{x}\|_{1}$, where $\mathbf{x}$ is the original signal. Since $A \hat{\mathbf{x}}=\mathbf{y}$, it easily follows that $\mathbf{w}=\hat{\mathbf{x}}-\mathbf{x}$ is in the null space of $A$. Therefore we can further write $\|\mathbf{x}\|_{1} \geq\|\mathbf{x}+\mathbf{w}\|_{1}$. Using the triangular inequality for the $l_{1}$ norm we obtain

$$
\begin{aligned}
\left\|\mathbf{x}_{K}\right\|_{1}+\left\|\mathbf{x}_{\bar{K}}\right\|_{1} & =\|\mathbf{x}\|_{1} \\
& \geq\|\hat{\mathbf{x}}\|_{1}=\|\mathbf{x}+\mathbf{w}\|_{1} \\
& \geq\left\|\mathbf{x}_{K}\right\|_{1}-\left\|\mathbf{w}_{K}\right\|_{1}+\left\|\mathbf{w}_{\bar{K}}\right\|_{1}-\left\|\mathbf{x}_{\bar{K}}\right\|_{1} \\
& \geq\left\|\mathbf{x}_{K}\right\|_{1}+\|\mathbf{w}\|_{1}-2\left\|\mathbf{w}_{K}\right\|_{1}-\left\|\mathbf{x}_{\bar{K}}\right\|_{1} \\
& \geq\left\|\mathbf{x}_{K}\right\|_{1}-\left\|\mathbf{x}_{\bar{K}}\right\|_{1}+\frac{C-1}{C+1}\|\mathbf{w}\|_{1}
\end{aligned}
$$

where the last two inequalities are from the claimed null-space property. Relating the first equality and the last inequality above, we finally get $2\left\|\mathbf{x}_{\bar{K}}\right\|_{1} \geq \frac{(C-1)}{C+1}\|\mathbf{w}\|_{1}$.

Necessity: Since every step in the proof of the sufficiency can be reversed if equality is achieved in the triangular equality, the condition $C \sum_{i=1}^{k}\left|\mathbf{w}_{K_{i}}\right| \leq \sum_{i=1}^{n-k}\left|\mathbf{w}_{\bar{K}_{i}}\right|$ is also a necessary condition for $\|\mathbf{x}-\hat{\mathbf{x}}\|_{1} \leq \frac{2(C+1)}{C-1}\left\|\mathbf{x}_{\bar{K}}\right\|_{1}$ to hold for every $\mathbf{x}$.

Remark:Of course, we need not to check (4) for all subsets $K$; checking the subset with the $k$ largest (in absolute value) elements of $\mathbf{w}$ is sufficient. However, Theorem 1 will be more convenient for our subsequent analysis.

It should also be noted that if the condition (4) is satisfied then $2\left\|\mathbf{x}_{\bar{K}}\right\|_{1} \geq \frac{(C-1)}{C+1}\|\mathbf{w}\|_{1}=\frac{(C-1)}{C+1}\|\hat{\mathbf{x}}-\mathbf{x}\|_{1}$ for any $K$ or $\bar{K}$. Hence it is also true for the set $K$ which corresponds to the $k$ largest components of the vector $\mathbf{x}$. In that case we can write $2 \delta \geq \frac{(C-1)}{C+1}\|\hat{\mathbf{x}}-\mathbf{x}\|_{1}$ which exactly corresponds to (3).

Now, let $Z$ be a basis of the null space of A, i.e. let $Z$ be a matrix such that $A Z=0$. Clearly, $Z$ is an $n \times(n-m)$ matrix. Furthermore, any $n \times 1$ vector $\mathbf{w}$ from the null-space of $A$ can be represented as $Z \mathbf{v}$ where $\mathbf{v} \in \mathbf{R}^{n-m}$. Then the condition from the Theorem 1 can be transformed to

$C \sum_{i=1}^{k}\left|Z_{K_{i}} \mathbf{v}\right| \leq \sum_{i=1}^{n-k}\left|Z_{\bar{K}_{i}} \mathbf{v}\right| \quad \forall \mathbf{v} \in \mathbf{R}^{n-m}, \forall K$ s. t. $|K|=k$

where $Z_{i}$ is the $i$-th row of the matrix $Z$. To facilitate writing let $I_{\mathbf{v}}$ denote the event $C \sum_{i=1}^{k}\left|Z_{K_{i}} \mathbf{v}\right| \leq \sum_{i=1}^{n-k}\left|Z_{\bar{K}_{i}} \mathbf{v}\right|$. In the following section we will for a given value $\alpha=\frac{m}{n}$, determine the value of $\beta=\frac{k}{n}$ such that (5) is satisfied with overwhelming probability.

The standard results on compressed sensing assume that the matrix $A$ has i.i.d. $\mathcal{N}(0,1)$ entries. In this case, the following lemma gives a characterization of the resulting null-space.

Lemma 1: Let $A \in R^{m \times n}$ be a random matrix with i.i.d. $\mathcal{N}(0,1)$ entries. Then the following statements hold:

- The distribution of $A$ is left-rotationally invariant, $P_{A}(A)=P_{A}(A \Theta), \Theta \Theta^{*}=\Theta^{*} \Theta=I$

- The distribution of $Z$, any basis of the null-space of $A$ is right-rotationally invariant. $P_{Z}(Z)=P_{Z}\left(\Theta^{*} Z\right), \Theta \Theta^{*}=$ $\Theta^{*} \Theta=I$ 
- It is always possible to choose a basis for the null-space such that $Z \in R^{n \times(n-m)}$ has i.i.d. $\mathcal{N}(0,1)$ entries.

In view of Theorem 1 and Lemma 1 what matters is that the null-space of $A$ be rotationally invariant. For any such $A$, the sharp bounds of ([1]), for example, apply. In this paper, we shall analyze the null-space directly. [It should be noted that we will present the result for the case when the matrix $Z$ has real Gaussian entries; however it is straightforward to extend it to the case when the matrix $Z$ is comprised of complex Gaussian entries.]

\section{Probabilistic ANALYSis OF THE NULl-SPACE CHARACTERIZATION}

In this section we probabilistically analyze the validity of (5). Before proceeding further, let us recall what exactly is the problem that we will solve in this section.

Assume that we are given an $n \times(n-m)$ matrix $Z$. Let $Z_{i}$ be the $i$-th row of $Z$ and let $Z_{i j}$ be the $i, j$-th element of $Z$. Further, let $Z_{i j}$ be i.i.d. zero-mean unit-variance Gaussian random variables. Let $\mathbf{v} \in \mathbf{R}^{n-m}$ be any real vector of length $(n-m)$. Let further $\alpha=\frac{m}{n}$ be a given constant independent of $n$. Then we will find $\beta=\frac{k}{n}$ such that

$$
\lim _{n \rightarrow \infty} P\left(I_{\mathbf{v}} \forall \mathbf{v} \in \mathbf{R}^{n-m}, \forall K \subset\{1,2, \ldots, n\},|K|=k\right)=1 .
$$

Proving (6) will of course be enough to prove that for all random matrices $A$ which have isotropically distributed nullspace, (2) with overwhelming probability solves (1). In order to prove (6) we will actually prove that

$$
\lim _{n \rightarrow \infty} P_{f}=0,
$$

$P_{f}=P\left(\exists \mathbf{v} \in \mathbf{R}^{n-m}, \exists K \subset\{1,2, \ldots, n\},|K|=k\right.$ s. t. $\left.\bar{I}_{\mathbf{v}}\right)$ and $\bar{I}_{\mathbf{v}}$ denotes the complement of $I_{\mathbf{v}}$, i.e. it denotes the event $C \sum_{i=1}^{k}\left|Z_{K_{i}} \mathbf{v}\right| \geq \sum_{i=1}^{n-k}\left|Z_{\bar{K}_{i}} \mathbf{v}\right|$. Now, using the union bound we can write

$P_{f} \leq \sum_{l=1}^{\left(\begin{array}{c}n \\ k\end{array}\right)} P\left(\exists \mathbf{v} \in \mathbf{R}^{n-m} \quad\right.$ s. t. $\left.\quad C \sum_{i=1}^{k}\left|Z_{K_{i}^{(l)}} \mathbf{v}\right| \geq \sum_{i=1}^{n-k}\left|Z_{\bar{K}_{i}^{(l)}} \mathbf{v}\right|\right)$

where $K^{(l)}$ is a subset of $\{1,2, \ldots, n\}$ and $\left|K^{(l)}\right|=k$. Clearly the number of these subsets is $\left(\begin{array}{l}n \\ k\end{array}\right)$ and hence the summation in (8) goes from 1 to $\left(\begin{array}{l}n \\ k\end{array}\right)$. Since the probability in (8) is insensitive to scaling of $\mathbf{v}$ by a constant we can restrict $\mathbf{v}$ to lie on the unit sphere (in $l_{2}$-norm). Furthermore, since the elements of the matrix $Z$ are i.i.d. all $\left(\begin{array}{l}n \\ k\end{array}\right)$ terms in the first summation on the right hand side of (8) will then be equal. Therefore we can further write

$P_{f} \leq\left(\begin{array}{c}n \\ k\end{array}\right) P\left(\exists \mathbf{v} \in \mathbf{R}^{n-m},\|\mathbf{v}\|_{2}=1\right.$ s. t. $\left.C \sum_{i=1}^{k}\left|Z_{i} \mathbf{v}\right| \geq \sum_{i=k+1}^{n}\left|Z_{i} \mathbf{v}\right|\right)$.

The main difficulty in computing the probability on the right hand side of (9) is in the fact that the vector $\mathbf{v}$ (i.e. its components) is continuous. Our approach will be based on the discrete covering of the unit sphere. In order to do that we will use small spheres of radius $\epsilon$. It can be shown [6],
[7], [4] that $\epsilon^{-(n-m)}$ spheres of radius $\epsilon$ is enough to cover the surface of the $(n-m)$-dimensional unit sphere. Let the coordinates of the centers of these $\epsilon^{-(n-m)}$ small spheres be the vectors $\mathbf{z}_{t}, t=1, \ldots, \epsilon^{-(n-m)}$. Clearly, $\left\|\mathbf{z}_{t}\right\|_{2}=\sqrt{1-\epsilon^{2}}$. Further, let $S_{t}, t=1, \ldots, \epsilon^{-(n-m)}$ be the intersection of the unit sphere and the hyperplane through $\mathbf{z}_{t}$ perpendicular to the line that connects $\mathbf{z}_{t}$ and the origin. It is not difficult to see that $\bigcup_{t=1}^{\epsilon^{-(n-m)}} S_{t}$ forms a body which completely encapsulates the origin. This effectively means that for any point $\mathbf{v}$ such that $\|\mathbf{v}\|>1$, the line connecting $\mathbf{v}$ and the origin will intersect $\bigcup_{t=1}^{\epsilon^{-(n-m)}} S_{t}$. Hence, we set $\mathcal{C}=\bigcup_{t=1}^{\epsilon^{-(n-m)}} S_{t}$ and apply union bound over $S_{t}$ to get

$P_{f} \leq\left(\begin{array}{l}n \\ k\end{array}\right) \epsilon^{-(n-m)} \max _{t}\left[P\left(\exists \mathbf{v} \in S_{t}: \frac{C \sum_{i=1}^{k}\left\|Z_{i} \mathbf{v}\right\|_{2}}{\sum_{i=k+1}^{n}\left\|Z_{i} \mathbf{v}\right\|_{2}} \geq 1\right)\right]$

Every vector $\mathbf{v} \in S_{t}$ can be represented as $\mathbf{v}=\mathbf{z}_{t}+\mathbf{e}$ where $\|\mathbf{e}\|_{2} \leq \epsilon$. Then we have

$$
\begin{aligned}
& \max _{t} P\left(\exists \mathbf{v} \in S_{t} \quad \text { s. t. } \quad C \sum_{i=1}^{k}\left|Z_{i} \mathbf{v}\right| \geq \sum_{i=k+1}^{n}\left|Z_{i} \mathbf{v}\right|\right) \\
= & \max _{t} P\left(\exists \mathbf{e},\|\mathbf{e}\|_{2} \leq \epsilon \text { s. t. } \frac{C \sum_{i=1}^{k}\left|Z_{i}\left(\mathbf{z}_{t}+\mathbf{e}\right)\right|}{\sum_{i=k+1}^{n}\left|Z_{i}\left(\mathbf{z}_{t}+\mathbf{e}\right)\right|} \geq 1\right) .
\end{aligned}
$$

Given the symmetry of the problem it should be noted that without loss of generality we can assume $\mathbf{z}_{t}=$ $\left[\left\|\mathbf{z}_{t}\right\|_{2}, 0,0, \ldots, 0\right]$. Further, using the results from [9] we have that $N^{n-m-1}$ points can be located on the sphere of radius $c \epsilon$ centered at $\mathbf{z}_{t}$ such that $S_{t}$ (whose radius is $\epsilon$ ) is inside a poly-tope determined by them and

$$
c \leq \begin{cases}\frac{1}{(1-\ln (N)) \sqrt{2 \ln (N)-\frac{\ln (n-m-1)}{n-m-1}}} & \text { if } N<\sqrt{2} \\ \frac{1}{1-\left(1+\frac{1}{N^{2}}\right) \frac{1}{2 N^{2}}} & \text { otherwise. }\end{cases}
$$

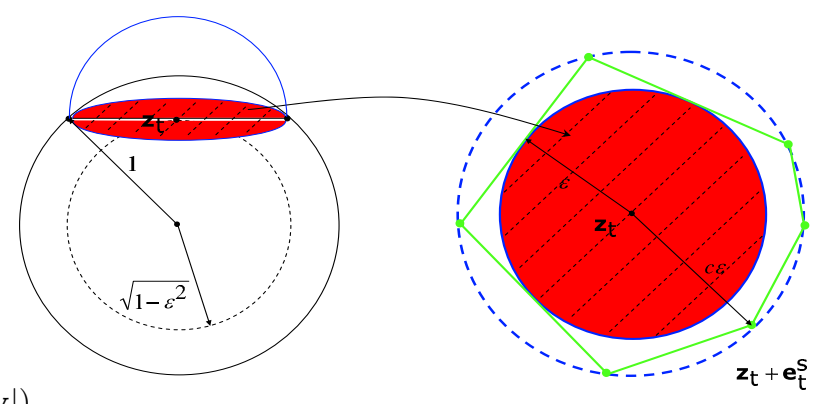

Fig. 1. Covering of the unit sphere

To get a feeling what values $N$ and $c$ can take we refer to [8] where it was stated that $3^{n-m-1}$ points can be located on the sphere of radius $\sqrt{\frac{9}{8}} \epsilon$ centered at $\mathbf{z}_{t}$ such that $S_{t}$ is inside a poly-tope determined by them. 
Let us call the poly-tope determined by $N^{n-m-1}$ points $P_{t}$. Let $\mathbf{e}_{t}^{s}, s=1,2, \ldots, N^{n-m}$ be its $N^{n-m-1}$ corner points. Since $\left|Z_{i} \mathbf{z}_{t}\right|-\left|Z_{i} \mathbf{e}\right| \leq\left|Z_{i}\left(\mathbf{z}_{t}+\mathbf{e}\right)\right|$, and $S_{t} \subset P_{t}$ we have

$$
\begin{aligned}
& \max _{t} P\left(\exists \mathbf{e},\left.|| \mathbf{e}\right|_{2} \leq \epsilon \quad \text { s. t. } \quad \frac{\sum_{i=k+1}^{n}\left|Z_{i}\left(\mathbf{z}_{t}+\mathbf{e}\right)\right|}{C \sum_{i=1}^{k}\left|Z_{i}\left(\mathbf{z}_{t}+\mathbf{e}\right)\right|} \leq 1\right) \\
\leq & \max _{t} P\left(\exists \mathbf{e},\left(\mathbf{z}_{t}+\mathbf{e}\right) \in P_{t} \text { s. t. } \frac{\sum_{i=k+1}^{n}\left(\left|Z_{i} \mathbf{z}_{t}\right|-\left|Z_{i} \mathbf{e}\right|\right)}{C \sum_{i=1}^{k}\left(\left|Z_{i} \mathbf{z}_{t}+Z_{i} \mathbf{e}\right|\right)} \leq 1\right) \\
\leq & \max _{t} P\left(\frac{\left.\sum_{i=k+1}^{n}\left|Z_{i} \mathbf{z}_{t}\right|-\max _{s} \sum_{i=k+1}^{n}\left|Z_{i} \mathbf{e}_{t}^{s}\right|\right)}{C \max _{s} \sum_{i=1}^{k}\left|Z_{i}\left(\mathbf{z}_{t}+\mathbf{e}_{t}^{s}\right)\right|} \leq 1\right) .
\end{aligned}
$$

where the second inequality follows from the property that the maximum of a convex function over a poly-tope is achieved at its corner points. Connecting (10), (11), and (13) we obtain

$P_{f} \leq \frac{\left(\begin{array}{l}n \\ k\end{array}\right)}{\epsilon^{(n-m)}} \max _{t} P\left(\frac{\left.\sum_{i=k+1}^{n}\left|Z_{i} \mathbf{z}_{t}\right|-\max _{s} \sum_{i=k+1}^{n}\left|Z_{i} \mathbf{e}_{t}^{s}\right|\right)}{C \max _{s} \sum_{i=1}^{k}\left|Z_{i}\left(\mathbf{z}_{t}+\mathbf{e}_{t}^{s}\right)\right|} \leq 1\right)$.

Using the union bound over $s$ we further have

$$
\begin{aligned}
& \max _{t} P\left(\frac{\sum_{i=k+1}^{n}\left|Z_{i} \mathbf{z}_{t}\right|-\max _{s} \sum_{i=k+1}^{n}\left|Z_{i} \mathbf{e}_{t}^{s}\right|}{C \max _{s} \sum_{i=1}^{k}\left|Z_{i}\left(\mathbf{z}_{t}+\mathbf{e}_{t}^{s}\right)\right|} \leq 1\right) \\
& \leq \max _{t} \sum_{s^{\prime}=1}^{N^{n-m}} P\left(\frac{\sum_{i=k+1}^{n}\left|Z_{i} \mathbf{z}_{t}\right|-\sum_{i=k+1}^{n}\left|Z_{i} \mathbf{e}_{t}^{s^{\prime}}\right|}{C \sum_{i=1}^{k}\left|Z_{i}\left(\mathbf{z}_{t}+\mathbf{e}_{t}^{s^{\prime}}\right)\right|} \leq 1\right)
\end{aligned}
$$

Given that only the first component of $\mathbf{z}_{t}$ is not equal to zero we can write
Let $G_{i}, F_{i}$ be independent zero-mean Gaussian random variables such that

$$
\operatorname{var}\left(G_{i}\right)=\left(\left\|\mathbf{z}_{t}\right\|_{2}-\left\|e_{t}^{s^{\prime}}\right\|_{2}\right)^{2}, \operatorname{var}\left(F_{i}\right)=\left\|\mathbf{e}_{t}^{s^{\prime}}\right\|_{2}^{2}, .
$$

Since $\operatorname{var}\left(F_{i}\right) \geq \operatorname{var}\left(D_{i}\right)$ and $\operatorname{var}\left(G_{i}\right) \leq \operatorname{var}\left(C_{i}\right)$ we have from (17)

$$
\begin{gathered}
N^{n-m-1} \max _{t, s^{\prime}} P\left(\sum_{i=1}^{k}\left|B_{i}\right|+\sum_{i=k+1}^{n}\left|D_{i}\right| \geq \sum_{i=k+1}^{n}\left|C_{i}\right|\right) \\
\leq N^{n-m-1} \max _{t, s^{\prime}} P\left(\sum_{i=1}^{k}\left|B_{i}\right|+\left|F_{i}\right| \geq \sum_{i=k+1}^{n}\left|G_{i}\right|\right) .
\end{gathered}
$$

Since $\left\|\mathbf{e}_{t}^{s^{\prime}}\right\|_{2}$ doesn't depend on $t, s^{\prime}$, the outer maximization can be omitted. Furthermore, $\left\|\mathbf{e}_{t}^{s^{\prime}}\right\|_{2}=c \epsilon$. Using the Chernoff bound we further have

$$
\begin{aligned}
& N^{n-m-1} P\left(\sum_{i=1}^{k}\left|B_{i}\right|+\sum_{i=k+1}^{n}\left|F_{i}\right| \geq \sum_{i=k+1}^{n}\left|G_{i}\right|\right) \\
& \quad \leq N^{n-m-1}\left(E e^{\mu\left|B_{1}\right|}\right)^{k}\left(E e^{\mu\left|F_{1}\right|}\right)^{n-k}\left(E e^{-\mu\left|G_{1}\right|}\right)^{n-k} .
\end{aligned}
$$

where $\mu$ is a positive constant. Connecting (14)-(19) we have $P_{f} \leq\left(\begin{array}{l}n \\ k\end{array}\right)\left(\frac{N}{\epsilon}\right)^{n-m}\left(E e^{\mu\left|B_{1}\right|} E e^{\mu\left|F_{1}\right|}\right)^{n-k}\left(E e^{-\mu\left|G_{1}\right|}\right)^{n-k}$.

After setting $k=\beta n, m=\alpha n$, and using the fact that $\left(\begin{array}{l}n \\ k\end{array}\right) \approx$ $e^{-n H(\beta)}$ we finally obtain we finally obtain

$\begin{aligned} & \max _{t} \sum_{s^{\prime}=1}^{N^{n-m-1}} P\left(\frac{\sum_{i=k+1}^{n}\left|Z_{i} \mathbf{z}_{t}\right|-\sum_{i=k+1}^{n}\left|Z_{i} \mathbf{e}_{t}^{s^{\prime}}\right|}{C \sum_{i=1}^{k}\left|Z_{i}\left(\mathbf{z}_{t}+\mathbf{e}_{t}^{s^{\prime}}\right)\right|} \leq 1\right) \leq N^{n-m-1} \times \\ & \max _{t, s^{\prime}} P\left(\frac{\sum_{i=k+1}^{n}\left(\left|Z_{i 1}\left(|| \mathbf{z}_{t} \|_{2}-\left|\left(\mathbf{e}_{t}^{s^{\prime}}\right)_{1}\right|\right)\right|-\left|\sum_{j=2}^{n-m} Z_{i j}\left(\mathbf{e}_{t}^{s^{\prime}}\right)_{j}\right|\right)}{C \sum_{i=1}^{k}\left|Z_{i}\left(\mathbf{z}_{t}+e_{t}^{s^{\prime}}\right)\right|} \leq 1\right.\end{aligned}$

where $\left(\mathbf{e}_{t}^{s^{\prime}}\right)_{j}$ denotes the $j$-th component of $\mathbf{e}_{t}^{s^{\prime}}$. Let $B_{i}=$ $C Z_{i}\left(\mathbf{z}_{t}+\mathbf{e}_{t}^{s^{\prime}}\right), 1 \leq i \leq k, C_{i}=Z_{i 1}\left(\left\|\mathbf{z}_{t}\right\|_{2}-\left|\left(\mathbf{e}_{t}^{s^{\prime}}\right)_{1}\right|\right), k+1 \leq$ $i \leq n$, and $D_{i}=\sum_{j=2}^{n=m} Z_{i j}\left(\mathbf{e}_{t}^{s^{\prime}}\right)_{j}, k+1 \leq i \leq n$. Clearly, $B_{i}$, $C_{i}, D_{i}$ are independent zero-mean Gaussian random variables. Furthermore it holds

$$
\begin{aligned}
& \operatorname{var}\left(B_{i}\right)=C^{2}\left\|\mathbf{z}_{t}+\mathbf{e}_{t}^{s^{\prime}}\right\|_{2}^{2}=1-\epsilon+c \epsilon^{2}, \\
& \operatorname{var}\left(C_{i}\right)=\left(\left\|\mathbf{z}_{t}\right\|_{2}-\left|\left(\mathbf{e}_{t}^{s^{\prime}}\right)_{1}\right|\right)^{2}, \operatorname{var}\left(D_{i}\right)=\left\|\mathbf{e}_{t}^{s^{\prime}}\right\|_{2}^{2}-\left|\left(\mathbf{e}_{t}^{s^{\prime}}\right)_{1}\right|^{2} .
\end{aligned}
$$

Then we can rewrite (16) as

$$
\begin{gathered}
\max _{t} \sum_{s^{\prime}=1}^{N^{n-m-1}} P\left(\frac{\sum_{i=k+1}^{n}\left|Z_{i} \mathbf{z}_{t}\right|-\sum_{i=k+1}^{n}\left|Z_{i} \mathbf{e}_{t}^{s^{\prime \prime}}\right|}{C \sum_{i=1}^{k}\left|Z_{i}\left(\mathbf{z}_{t}+\mathbf{e}_{t}^{s^{\prime}}\right)\right|} \leq 1\right) \leq \\
N^{n-m-1} \max _{t, s^{\prime}} P\left(\sum_{i=1}^{k}\left|B_{i}\right|+\sum_{i=k+1}^{n}\left|D_{i}\right| \geq \sum_{i=k+1}^{n}\left(\left|C_{i}\right|\right)\right) .
\end{gathered}
$$

$$
\lim _{n \rightarrow \infty} P_{f} \leq \lim _{n \rightarrow \infty} \xi^{n}
$$

where

$$
\begin{gathered}
\xi=\frac{(N / \epsilon)^{(1-\alpha)}}{e^{H(\beta)}}\left(e^{\mu^{2} R_{u}^{2} / 2}\left(\operatorname{erf}\left(\mu \frac{R_{u}}{\sqrt{2}}\right)+1\right)\right)^{\beta} \times \\
\left(e^{\mu^{2} R_{s}^{2} / 2}\left(\operatorname{erf}\left(\frac{\mu R_{s}}{\sqrt{2}}\right)+1\right)\right)^{1-\beta}\left(e^{\mu^{2} R_{l}^{2} / 2} \operatorname{erfc}\left(\mu \frac{R_{l}}{\sqrt{2}}\right)\right)^{(1-\beta)}
\end{gathered}
$$

$R_{u}=C \sqrt{1-\epsilon^{2}+(c \epsilon)^{2}}, R_{l}=\sqrt{1-\epsilon^{2}}-c \epsilon$, and $R_{s}=c \epsilon$.

Using well known bounds on the erf and erfc function it can easily be shown that for any value of $\alpha$ there will be a constant (independent of $n$ ) value for $\beta$ such that $\xi<1$.

We summarize the previous results in the following theorem.

Theorem 2: Assume that we use (2) to approximate the solution of (1). Further assume that the matrix $A$ in (1) has isotropically distributed null-space and that the number of rows of the matrix $A$ is $m=\alpha n$. Let $\hat{\mathbf{x}}$ be the solution of (2). Then $\hat{\mathbf{x}}$ approximates the vector $\mathbf{x}$ from (1) such that (3) holds provided that $\mathbf{x}$ has more than $(1-\beta) n$ small components which form a vector with $l_{1}$ norm smaller than a constant $\delta$. $\alpha$ and $\beta$ are absolute constants and independent of $n$. Furthermore, for any given $\alpha$ the explicit value of $\beta$ can be numerically determined as a maximal value of $\beta$ so that the right hand side of (22) is less than 1 . 
Proof: Follows from the previous discussion.

To get a feeling what values of sparsity $\beta$ are achievable we should find the maximal allowable value for $\beta$ such that for a given value of $\alpha, \xi<1$. In the following section we discuss numerical results shown on Figure 2.

\section{NUMERICAL RESULTS}

As we have said above, for a given value of $\alpha$ numerical optimization of $\xi$ over $N, c, \epsilon, 0<\beta \leq \frac{\alpha}{2}$ will produce the range of $\beta$ such that $\xi<1$. This range of $\beta$ is allowable sparsity so that (2) approximates solution of (1) as stated in (3) with overwhelming probability over the choice of the measurement matrices $A$ for any given approximately sparse vector $\mathbf{x}$.
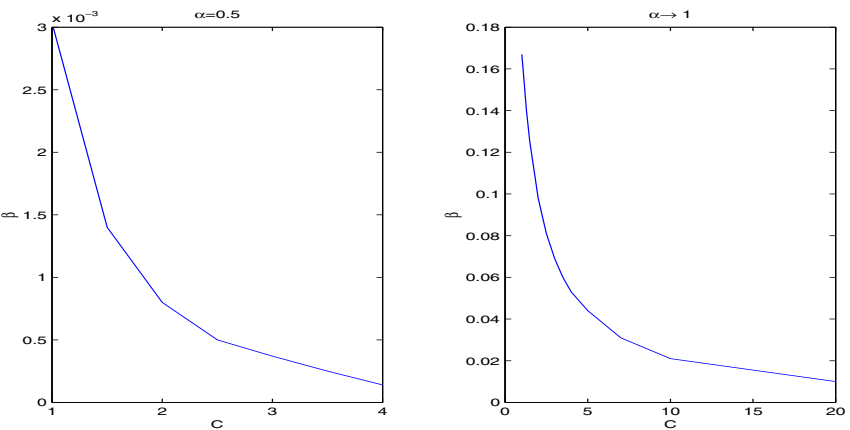

Fig. 2. Allowable sparsity as a function of $C$ (allowable imperfection of the recovered signal is $\frac{2(C+1) \delta}{C-1}$ )

On Figure 2 we show allowable sparsity $\beta$ as a function of parameter $C$ for 2 different values of $\alpha$. Namely, we consider the case $\alpha=0.5$ and the asymptotic case $\alpha \longrightarrow 1$. It is interesting to note that in the asymptotic case $\alpha \longrightarrow 1$ as $C \longrightarrow 1$ (perfectly sparse case), we obtain $\beta \longrightarrow 0.168$ which corresponds to the result of [1] obtained in the perfectly sparse case. We should mention that given the fact that we are using the union bound over all possible $k$ locations of the high components of $\mathbf{x}$ this result as well as result of [1] is a lower bound to the optimal value of $\beta$. This lower bound could be improved to the exact value $\beta=0.23$ if instead of simple union bounding we used a bit more sophisticated sorting idea from [15].

\section{CONCLUSION}

We analyzed a null-space characterization of the necessary and sufficient conditions for the success of the $l_{1}$-norm optimization in compressed sensing of the approximately sparse signals. Our analysis provided a somewhat new technique in proving the optimality of the $l_{1}$-norm optimization for measurement matrices with isotropically distributed null-space.

\section{ACKNOWLEDGMENT}

This work was supported in part by the National Science Foundation under grant no. CCR-0133818, by the David and Lucille Packard Foundation, and by Caltech's Lee Center for Advanced Networking.

\section{REFERENCES}

[1] David Donoho and Jared Tanner, "Neighborliness of randomly-projected simplices in high dimensions", Proc. National Academy of Sciences, 102(27), pp. 9452-9457, 2005.

[2] Emmanuel Candes and Terence Tao, "Decoding by linear programming", IEEE Trans. on Information Theory, 51(12), pp. 4203 - 4215, December 2005.

[3] Richard Baraniuk, Mark Davenport, Ronald DeVore, and Michael Wakin, "A simple proof of the restricted isometry property for random matrices", To appear in Constructive Approximation, available online at http://www.dsp.ece.rice.edu/cs/.

[4] Mark Rudelson and Roman Vershynin, "Geometric approach to error correcting codes and reconstruction of signals", International Mathematical Research Notices, 64, pp. 4019 - 4041, 2005.

[5] A.M. Vershik and P.V. Sporyshev, "Asymptotic Behavior of the Number of Faces of Random Polyhedra and the Neighborliness Problem", Selecta Mathematica Sovietica vol. 11, No. 2 (1992).

[6] A.D. Wyner, "Random packings and coverings of the unit N-sphere", Bell systems technical journal, vol. 46, 2111-2118, 1967.

[7] I. Dumer, M.S. Pinsker, and V.V. Prelov,"On the thinest coverings of the spheres and ellipsoids", Information Transfer and Combinatorics, LNCS 4123, pp.883-910, 2006.

[8] I. Barany and N. Simanyi, "A note on the size of the largest ball inside a convex polytope", Periodica Mathematica Hungarica, vol. 51 (2), 2005, pp. 15-18.

[9] K. Boroczky, Jr. and G. Wintsche, "Covering the sphere by equal spherical balls", in Discrete and computational geometry: the Goodman-Pollach Festschrift (ed. by S. Basu et al.), 2003, 237-253.

[10] A. Feuer and A. Nemirovski, "On sparse representation in pairs of bases", IEEE Transactions on Information Theory 49(6): 1579-1581 (2003)

[11] N. Linial and I. Novik, "How neighborly can a centrally symmetric polytope be?", Discrete and Computational Geometry, 36(2006) 273-281.

[12] Y. Zhang, "When is missing data recoverable", available online at http://www.dsp.ece.rice.edu/cs/.

[13] J. Haupt and R. Nowak, "Signal reconstruction from noisy random projections", IEEE Trans. on Information Theory, 52(9), pp. 4036-4048, September 2006.

[14] M. J. Wainwright, "Sharp thresholds for high-dimensional and noisy recovery of sparsity", Proc. Allerton Conference on Communication, Control, and Computing, Monticello, IL, September 2006.

[15] C. Dwork, F. McSherry, and K. Talwar, "The price of privacy and the limits of LP decoding", Symp. on Theory of Computing (STOC), San Diego, California, June, 2007.

[16] A. Cohen, W. Dahmen, and R. DeVore, "Compressed sensing and best k-term approximation". (Preprint, 2006), available online at http://www.dsp.ece.rice.edu/cs/

[17] J. Tropp, M. Wakin, M. Duarte, D. Baron, and R. Baraniuk, Random filters for compressive sampling and reconstruction. Proc. IEEE Int. Conf on Acoustics, Speech, and Signal Processing (ICASSP), Toulouse, France, May 2006.

[18] Emmanuel Candes, "Compressive sampling", Proc. International Congress of Mathematics, 3, pp. 1433-1452, Madrid, Spain, 2006. 